\title{
Safety of Prolonged Dexmedetomidine Use in Pediatric Patients
}

\author{
Jennifer T. Wintergrass (PharmD), Anna Simmont (PharmD, BCPS), Catherine Chen (PharmD, BCPS) and \\ Kimberly Leuthner (PharmD, fIDSA)
}

Department of Pharmacy, University Medical Center of Southern Nevada

\begin{abstract}
How to cite this paper: Wintergrass, J. T., Simmont, A., Chen, C., Leuthner, K. (2020) Safety of Prolonged Dexmedetomidine Use in Pediatric Patients. International Journal of Clinical and Experimental Medicine Research, 4(1), 135-140

DOI: $10.26855 /$ ijcemr.2020.03.001
\end{abstract}

Corresponding author: Jennifer T. Wintergrass, PharmD, Department of Pharmacy, 1800W Charleston Blvd, Las Vegas, NV 89102.

Email: jennifer.wintergrass@umcsn.com

\begin{abstract}
We evaluated the incidence of hemodynamic adverse events in children who received prolonged continuous dexmedetomidine for $\geq 192$ hours. Prolonged infusions of dexmedetomidine may additionally contribute to hemodynamic adverse events at time of discontinuation. The current practices regarding use of dexmedetomidine at time of dexmedetomidine discontinuation were also evaluated. This was a retrospective, chart review of 14 children who had received prolonged continuous dexmedetomidine infusions. Demographics, dexmedetomidine infusion characteristics, systolic and diastolic blood pressure, heart rate, use of rescue medication, and clonidine use characteristics were evaluated during dexmedetomdine infusion through 24-hr after last dose of dexmedetomidine. Average age was 5 years (range 7 months -17 years). Average length of continuous dexmedetomidine was 10 days (range $8-19$ days). Average dose of dexmedetomidine during initiation and maintenance was $1.25 \mathrm{mcg} / \mathrm{kg} / \mathrm{hr}$. The maximum rate of dexmedetomidine was $1.6 \mathrm{mcg} /$ $\mathrm{kg} / \mathrm{hr}$. Statistically significant higher adverse event incidence rate seen during dexmedetomidine wean compared to initiation and maintenance $(36.2 \%$ and $0.04 \%, p<0.05)$. No differences were seen in rate of adverse events comparing patients who were weaned with and without clonidine $39.9 \%$ and $37.8 \%, \mathrm{p}=0.52$ ). Prolonged continuous infusions of dexmedetomidine were well-tolerated with doses up to $1.6 \mathrm{mcg} / \mathrm{kg} / \mathrm{hr}$. A higher incidence of adverse events were seen during the period of wean compared to period of initiation and maintenance. Clonidine use at wean was not found to have an effect on decreasing the amount of hemodynamic adverse events at discontinuation.
\end{abstract}

\section{Keywords}

pediatric, dexmedetomidine, prolonged, safety

\section{Introduction}

Dexmedetomidine is a central-acting selective $\alpha 2$-agonist producing both anesthetic and sedative properties. Currently FDA-approved for use in adults for intensive care unit (ICU) sedation in patients with mechanical ventilation as a continuous infusion (up to 24 hours) and for procedural sedation, limited safety and efficacy data exists regarding ICU or procedural sedation in pediatric patients. ${ }^{[1]}$ The off-label use of dexmedetomidine for sedation in the pediatric population has increased due to the potential opioid and benzodiazepine sparing effects of dexmedetomidine and lack of clinically significant respiratory depression. ${ }^{[2-4]}$ As further experience is gained regarding the use of dexmedetomidine in pediatric critical care, published data supporting durations of use longer than 24 hours remains limited.

Both dexmedetomidine and clonidine are centrally acting $\alpha_{2}$-agonists which have been evaluated as alternatives to traditional agents for sedation due to their sedative, analgesic, and anxiolytic properties. When $\alpha_{2}$-receptors are activated centrally, the result is suppression of the sympathetic nervous system. Long-term use of clonidine has been 
documented to cause withdrawal symptoms upon discontinuation. ${ }^{[5]}$ Clonidine withdrawal symptoms are seen due to increased sympathetic activity as a result of loss in central $\alpha_{2}$-receptor activation. ${ }^{[5]}$ Dexmedetomidine and clonidine are structurally similar; however, dexmedetomidine has an $\alpha_{2}: \alpha_{1}$ specificity ratio of 1600:1 compared to 200:1 for clonidine. ${ }^{[5]}$ Although not well documented, dexmedetomidine has the potential to cause withdrawal symptoms similar to those of clonidine. There are currently no recommendations regarding procedures for weaning dexmedetomidine after extended durations of use, however clonidine has been used anecdotally.

One of the earliest reports of prolonged use of dexmedetomidine in the pediatric population is a case report of a 9 year-old boy who received a continuous infusion of dexmedetomidine for 4 days ${ }^{[6]}$ No clinical signs of withdrawal or rebound hypertension were noted upon time of dexmedetomidine discontinuation. In a retrospective review, Reiter et al. evaluated the efficacy and tolerability of dexmedetomidine infusions in 29 patients. Approximately one-third of patients received a loading dose upon initiation. ${ }^{[3]}$ The mean infusion time was $110 \pm 83$ hours (range 32-378). ${ }^{[3]} \mathrm{A}$ statistically significant decrease in heart rate was seen at time of dexmedetomidine initiation, however, no significant changes in hemodynamics were noted throughout period of continued use. ${ }^{[3]}$ Bejian et al. performed an evaluation of 54 pediatric cardiovascular ICU (CVICU) patients who received prolonged continuous dexmedetomidine infusions. The mean duration of dexmedetomidine use of 37.3 hours (range 2-177). ${ }^{[7]}$ No clinically significant changes in hemodynamic parameters, including mean arterial pressure and heart rate, were noted during or after use of dexmedetomidine. Gupta et al. also performed a retrospective review in pediatric CVICU patients, but included patients who had received dexmedetomidine infusions for greater than or equal to 96 hours ${ }^{[4]}$ The median duration of use was 196 hours. When compared to the control group, there was no statistically significant difference seen in either heart rate or blood pressure during period of dexmedetomidine infusion. ${ }^{[4]} \mathrm{A}$ study done by Whalen et al. evaluated the use of long-term dexmedetomidine in 98 critically ill children and neonates. ${ }^{[8]}$ Median duration of dexmedetomidine use was 141 hours. ${ }^{[8]}$ After discontinuation of dexmedetomidine, systolic blood pressure (SBP), diastolic blood pressure (DBP), and heart rate (HR) were found to be statistically higher than baseline. ${ }^{[8]}$ Of the 26 patients with recorded withdrawal scores, the most common symptoms of withdrawal included agitation (65\%), decreased sleep (54\%), and tremors $(38 \%)$ at the time of dexmedetomidine discontinuation. ${ }^{[8]}$

Lardieri et al. evaluated the presence withdrawal symptoms in pediatric patients receiving clonidine versus those without clonidine while weaning off dexmedetomidine. ${ }^{[9]}$ A statistically significant difference was seen in the average heart rate and mean change in heart rate postwean in patients who had received clonidine versus patients that did not. ${ }^{[9]}$ No statistically significant difference in WAT-1 scores was seen, however the clonidine group trended towards having lower WAT-1 scores during the 24 hours post dexmedetomidine wean. ${ }^{[9]}$

The previously mentioned trials were small, lacked uniformity in patient population, and included variable durations of dexmedetomidine therapy. Conflicting data exists as to whether prolonged continuous infusions of dexmedetomidine result in a higher risk of hemodynamic adverse events. The purpose of this study is to evaluate the hemodynamic safety of dexmedetomidine infusions 8 days (192 hours) or longer. Hemodynamic safety was determined by the presence of hypotension and/or bradycardia during period of initiation and maintenance and by the presence of hypertension and/or tachycardia during the period of dexmedetomidine wean. We also hope to identify patterns in our current procedures for discontinuation of prolonged dexmedetomidine infusions. In addition, a subgroup analysis will be performed on patients weaned with and without clonidine to determine the ability of clonidine to attenuate hemodynamic adverse effects or withdrawal symptoms.

\section{Materials and Methods}

This was a retrospective, single-center study evaluating the use of prolonged continuous dexmedetomidine (DEX) use for 8 days (192 hours) or longer in pediatric patients. Institutional review board approval was obtained prior to beginning of the study. All patients under 18 years of age with DEX use greater than or equal to 192 hours between March 1, 2013 to September 30, 2016 were reviewed for inclusion into our study. Patients with DEX infusions stopped for 12 hours or greater prior to reaching 192 hours of use were excluded. Patients were also excluded if there was missing documentation regarding the DEX infusion.

The primary objective was to determine the prevalence of hemodynamic adverse events throughout prolonged continuous DEX use. For the period of DEX initiation and maintenance, hemodynamic adverse events were defined as episodes of hypotension and bradycardia. Hypotension was defined as a systolic blood pressure (SBP) $<70 \mathrm{mmHg}$ for infants, $\mathrm{SBP}<70+$ [age (yr) x 2] $\mathrm{mmHg}$ for children ages $1-9$, and $\mathrm{SBP}<90 \mathrm{mmHg}$ for adolescents ages $\geq 10$. 
Bradycardia was defined as a heart rate (HR) $<90$ BPM for infants, HR $<80$ BPM for children ages $1-2$, HR $<65$ BPM for children age $3-5$, HR $<58$ BPM for children ages $6-11$, and HR $<50$ BPM for adolescents ages $\geq 12$. For the period of DEX wean, hemodynamic adverse events were defined as episodes of hypertension and tachycardia. Hypertension was defined as a SBP and/or diastolic blood pressure (DBP) $\geq 95^{\text {th }}$ percentile for patients' gender, age, and height. Tachycardia was defined as a HR $>160$ BPM for infants, HR $>140$ BPM for children age 2 to 12 , and HR $>100$ BPM for adolescents age $\geq 13$ years of age. The period of the dexmedetomidine wean was defined as the period of dexmedetomidine down titration as indicated by any of the following: written note in the physicians' progress notes, note in the dexmedetomidine medication order, or the time of first dose decrease with no further increase in a 12-hour period. We also recorded if rescue medications were used in response to a hemodynamic adverse event including the use of vasopressors, atropine, fluid bolus, or inotropes during the period of initiation and maintenance and the usage of antihypertensives or adenosine during period of wean.

The secondary objective was to assess whether clonidine use during wean of prolonged continuous dexmedetomidine had effects on Withdrawal Assessment Tool-1 (WAT-1) scores or incidence of hemodynamic adverse events. WAT-1 scores were recorded by the bedside nurse every 12 hours or more frequently as indicated by the physician to determine presence of withdrawal symptoms. The WAT-1 scoring includes presence of the following: loose or watery stools; vomiting, retching, or gagging; temperature $>37.8^{\circ} \mathrm{C}$; pre-stimulus observation of patient state, tremor, sweating, uncoordinated or repetitive movement, yawning or sneezing; stimulus observation of startle in response to touch, muscle tone; post-stimulus recover of time to gain calm state. WAT- 1 scores range from 0 to 12 , with a score $\geq 3$ indicative of withdrawal.

The following data were collected: patient demographics, duration of dexmedetomidine, dexmedetomidine dose at initiation, max dexmedetomidine dose, time at hemodynamic adverse event, dexmedetomidine dose at hemodynamic adverse event, type of hemodynamic adverse event, use of rescue medication at time of hemodynamic adverse event, initial clonidine dose, maximum clonidine dose, duration of clonidine use, duration of dexmedetomidine prior to clonidine start, and WAT-1 scores during dexmedetomidine wean. Patients were followed until 24 hours post last administration of dexmedetomidine. Descriptive statistics were used to characterize the population, dexmedetomidine use, and clonidine use. Chi-squared tests were used to analyze categorical variables. Regression analysis was used to determine correlation between variables.

\section{Results}

Twenty-seven patients were identified as having received dexmedetomidine for at least 192 hours. Thirteen patients were excluded, 12 patients for non-continuous dexmedetomidine usage prior to reaching 192 hours and 1 patient with missing documentation regarding dexmedetomidine infusion. Fourteen patients were enrolled in this study.

The mean age of patients was 4.9 years of age (range 0.6-17) (Table 1). Six of 14 patients were male (40.0\%). The primary diagnosis was acute respiratory failure in 4 patients $(27.0 \%)$, pneumonia in 3 patients $(20.0 \%)$, burn in 2 patients (13.0\%), and 5 other (40.0\%). Mean PICU length of stay was 43.1 days (range 15-105). Mean duration of mechanical ventilation was 21.9 days (range 8-46). Mean duration of continuous dexmedetomidine use was 10.1 days (range 8-18.8).

\section{Table 1. Patient Demographics}

\begin{tabular}{ll}
\hline Age, yr (range) & $4.9(0.6-17)$ \\
Male, n (\%) & $6(40.0)$ \\
& $4(27.0)$ acute respiratory failure \\
Primary diagnosis, n (\%) & $3(20.0)$ pneumonia \\
& $2(13.0)$ burn \\
PICU length of stay, days (range) & $5(40.0)$ other* \\
Mechanical ventilation, days (range) & $43.1(15-105)$ \\
Duration of dexmedetomidine use, days (range) & $21.9(8-46)$ \\
\hline
\end{tabular}

*one patient with one of the following primary diagnoses: foreign object asphyxiation, stenosis of larynx, craniosynotosis, cholelithiasis, mechanical ventilator breakdown, CSF leak 
The mean duration of dexmedetomidine during initiation and maintenance was 8.1 days (range 3.6-15.7) (Table 2). Mean initial rate of dexmedetomidine was $0.5 \mathrm{mcg} / \mathrm{kg} / \mathrm{hr}$ (range $0.4-0.8$ ). Doses of dexmedetomidine were titrated to a mean maximum of $1.3 \mathrm{mcg} / \mathrm{kg} / \mathrm{hr}$ (range $0.7-1.6$ ). Four of 14 patients (28.6\%) experienced at least 1 hemodynamic adverse event during the period of initiation and maintenance. No patients in our study received loading doses of dexmedetomidine at initiation. A total of 11 hemodynamic adverse events were seen ( 9 episodes of hypotension and 2 episodes of bradycardia) resulting in an adverse event rate of $0.4 \%$. At time of hemodynamic adverse event, the mean dose of dexmedetomidine was $1.2 \mathrm{mcg} / \mathrm{kg} / \mathrm{hr}$ (range $0.8-1.6$ ). Only 1 adverse event required use of rescue medication ( $250 \mathrm{~mL}$ LR bolus) representing $9.1 \%$ of all hemodynamic adverse events experienced during this time. The duration of dexmedetomidine initiation and maintenance was not found to be correlated to the incidence of hemodynamic adverse events (Figure 1).

Table 2. Dexmedetomidine Use

\begin{tabular}{lll}
\hline & Initiation and maintenance & Wean \\
\hline Duration, days (range) & $8.1(3.6-15.7)$ & $1.9(0.04-5.1)$ \\
Starting rate, $\mathrm{mcg} / \mathrm{kg} / \mathrm{hr}$ (range) & $0.5(0.4-0.8)$ & $1.1(0.4-1.6)$ \\
Maximum rate, $\mathrm{mcg} / \mathrm{kg} / \mathrm{hr}$ (range) & $1.3(0.7-1.6)$ & $\mathrm{N} / \mathrm{A}$ \\
Patients with $\geq 1$ adverse event, $\mathrm{n}(\%)$ & $4(28.6)$ & $14(100.0)$ \\
Adverse event incidence rate & $0.4 \%$ & $36.2 \% *$ \\
DEX at time of adverse event, $\mathrm{mcg} / \mathrm{kg} / \mathrm{hr}$ & $1.2(0.8-1.6)$ & $0.33(0-1.3)$ \\
Events requiring rescue medication, $\mathrm{n}(\%)$ & $1(9.1)$ & $5(1.4)$ \\
\hline * $<<0.05$ & &
\end{tabular}

The mean duration of dexmedetomidine during wean was 1.9 days (range 0.04-5.1)(Table 2). Mean dose of dexmedetomidine at start of wean was $1.1 \mathrm{mcg} / \mathrm{kg} / \mathrm{hr}$ (range 0.4-1.6). Ten of $14(72.0 \%)$ patients were given clonidine at the start of dexmedetomidine wean. All 14 patients experienced at least 1 hemodynamic adverse events during dexmedetomidine wean. A total of 369 hemodynamic adverse events were seen (154 episodes of hypertension, 161 episodes of tachycardia, and 54 episodes of hypertension \& tachycardia) resulting in an adverse event rate of $36.2 \%$. A statistically significant higher adverse event rate was seen in the period of dexmedetomidine wean versus initiation and maintenance $(\mathrm{p}<0.05)$. At time of hemodynamic adverse event, the mean dose of dexmedetomidine was $0.33 \mathrm{mcg} /$ $\mathrm{kg} / \mathrm{hr}$ (range $0-1.3$ ). 39\% of adverse events occurred during the 24-hours post last dose of dexmedetomidine. Rescue medications were used during $5(1.4 \%)$ adverse events including 4 uses of labetalol and 1 use of chlorothiazide. The duration of dexmedetomidine wean was not found to be correlated to the incidence of hemodynamic adverse events (Figure 1).

Of the 10 patients given clonidine at time of dexmedetomidine wean, $9(90.0 \%)$ received a $0.1 \mathrm{mg}$ patch and $1(10.0 \%)$ received a $0.2 \mathrm{mg}$ patch. Three (27.0\%) patients remained on $0.1 \mathrm{mg}$ patch during dexmedetomidine wean, $5(46.0 \%)$ were titrated up to the $0.2 \mathrm{mg}$ patch, $2(18.0 \%)$ patients were titrated up to the $0.3 \mathrm{mg}$ patch, and $1(9.0 \%)$ patient was transitioned to an oral clonidine regimen. Mean duration of clonidine use was 14.9 days (range 9.2-22.3). Two patients were excluded from this analysis of duration of use as they continued clonidine between separate continuous dexmedetomidine infusions greater then 192 hours. The mean duration of dexmedetomidine prior to clonidine start was 7.7 days (range 1.6-13). An average 3.05 days of overlap was seen between clonidine start and last dose of dexmedetomidine (range -0.1-8.5). No significant difference in adverse event incidence rate was seen in patients that did not receive clonidine versus patients that received clonidine at start of dexmedetomidine wean $(39.9 \%$ vs. $37.8 \%$, $\mathrm{p}=0.52$ ). 

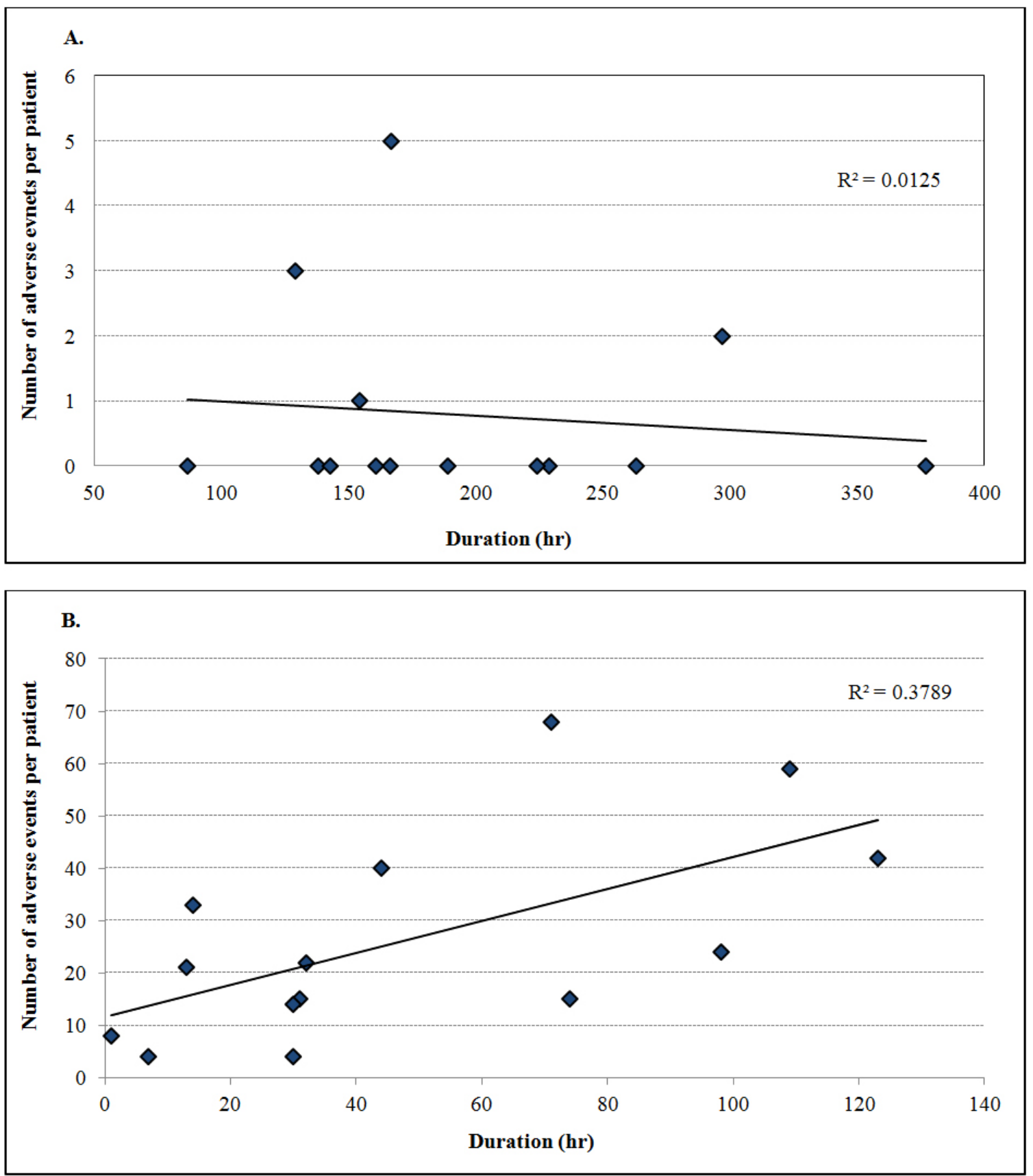

Figure 1. Correlation between length of initiation and maintenance phase and adverse events (A.) and correlation between length of wean and adverse events (B.)

\section{Discussion}

This study was intended to determine whether prolonged continuous infusions of dexmedetomidine were safe in pediatric populations using incidence of hemodynamic adverse events. The overall incidence rate of hemodynamic adverse events was low during period of dexmedetomidine initiation and maintenance and dexmedetomidine wean. A higher incidence of hemodynamic adverse events was noted during the wean, however a limited number of adverse events required use of a rescue medication. As vitals are recorded hourly for PICU patients, there may be reporting bias in determining the frequency of hemodynamic adverse events. Very few of the hemodynamic adverse events may have been clinically significant as the overall use of rescue mediation was low. Previous studies evaluating hemodynamic 
adverse events with dexmedetomidine use found prolonged infusions to be well tolerated, with potential for more adverse events at time of dexmedetomidine wean. The results of this study are consistent with previous studies in that the period of dexmedetomidine wean may see higher incidence of hemodynamic adverse events.

There were no differences seen in the incidence of hemodynamic adverse events at dexmedetomidine wean in the patients that had received clonidine compared to patients that did not receive clonidine at wean. In our study, we did not find clonidine able to attenuate hemodynamic adverse events during dexmedetomidine wean. The study by Lardieri et al. found clonidine to be beneficial in decreasing the degree of hemodynamic changes at time of dexmedetomidine wean. In our study, describing the use of clonidine was a secondary objective and larger number of included patients may be needed to be able to detect a statistically significant difference.

This study does have limitations including the retrospective nature, limited number of included patients, and inability to perform analyses on WAT-1 scores due to missing data. The WAT-1 scores were commonly not within the permanent chart or taken within the time frame being evaluated. WAT-1 scores are most typically used for assessing withdrawal from opiates or benzodiazepines, therefore WAT-1 score may not have been performed at the time of dexmedetomidine wean unless other medications were also being titrated down simultaneously. Clonidine may also have been used for the prevention of hemodynamic adverse effects due to the down titration of opioids or benzodiazepines and not solely for dexmedetomidine.

In this retrospective analysis, a statistically significant increase in the incidence rate of hemodynamic adverse events were seen during the period of dexmedetomidine wean compared to the period of dexmedetomidine initiation and maintenance. Overall, prolonged continuous infusions of dexmedetomidine 192 hours or longer were well tolerated with doses ranging up to $1.6 \mathrm{mcg} / \mathrm{kg} / \mathrm{hr}$. Clonidine was not found to be beneficial at time of dexmedetomidine wean in preventing hemodynamic adverse events. Additional studies are needed with a larger sample size to determine whether prolonged infusions of dexmedetomidine are safe in pediatric patients.

\section{References}

[1] Precedex® [package insert]. Lake Forest, IL: Hospira, Inc; 2016.

[2] Belleville JP, Ward DS, Bloor BC, Maze M. Effects of intravenous dexmedetomidine in humans. Sedation, ventilation, and metabolic rate. Anesthesiology. 1992; 77:1125-33.

[3] Reiter PD, Pietras M, Dobyns EL. Prolonged dexmedetomidine infusions in critically ill infants and children. Indian Pediatr. 2009; 46: 767-73.

[4] Gupta P, Whiteside W, Sabati A et al. Safety and efficacy of prolonged dexmedetomidine use in critically ill children with heart disease. Pedriatr Crit Care Med. 2012; 150:660-6.

[5] Virtanen R, Savola JM, Saano V et al. Characterization of selectivity, specificity and potency of dexmedetomidine as alpha-2-adrenoreceptor agonist. Eur J Pharmacol. 1988; 150:9-14.

[6] Hammer GB, Phillip BM, Schroeder AR et al. Prolonged infusion of dexmedetomidine for sedation following tracheal resection. Paediatr Anaesth. 2005; 15:616-20.

[7] Bejian S, Valasek C, Nigro JJ et al. Prolonged use of dexmedetomidine in the paediatric cardiothoracic intensive care unit. Cardiol Young. 2009; 19: 98-104.

[8] Whalen LD, Di Gennaro JL, Irby GA et al. Long-term dexmedetomidine use and safety profile among critically ill children and neonates. Pediatr Crit Care Med. 2014; 15: 706-14.

[9] Lardieri AB, Fusco NM, Simone S et al. Effects of clonidine on withdrawal from long-term dexmedetomidine in the pediatric patient. J Pediatr Pharmacol Ther. 2015; 20:45-53. 